\title{
Auto-seqüências da Transformada Numérica de Fourier
}

\author{
H. M. Cavalcanti, R. M. Campello de Souza
}

\begin{abstract}
Resumo-Sinais cujas formas não variam quando submetidos à Transformada Numérica de Fourier (TNF) e que geram uma classe de autofunções do operador unitário TNF são apresentados. A utilização de tais seqüências invariantes como assinaturas de usuários em um sistema de comunicação multiusuário através do Canal Somador Real (t-RAC) é sugerida.
\end{abstract}

Palavras-Chave-Transformadas numéricas, comunicação multiusuário, canal somador real, transformada de Fourier de corpo finito.

Abstract-shape-invariant signals under the Number Theoretic Fourier Transform (NTFT) are investigated, leading to a class of eigenfunctions for the unitary NTFT operator. Such invariant sequences (eigensequences) are suggested as user signatures over the real adder channel (t-RAC) for a multiuser communication system.

Keywords-Number theoretic transforms, multiuser comunication, real adder channel, finite field Fourier transform.

\section{INTRODUÇÃO}

Este trabalho investiga as auto-sequiências do operador Transformada Numérica de Fourier (TNF). Uma seqüência cuja forma não é alterada pelo operador TNF é uma seqüência invariante à TNF (SITNF). Tais sequiências são importantes em aplicações envolvendo análise de espectro, já que a complexidade computacional de suas TNFs é linear. Por ser uma transformada de corpo finito, a TNF oferece ainda uma vantagem a mais em relação à Transformada Discreta de Fourier (DFT), que é o fato de só se trabalhar com números inteiros, evitando assim problemas com arredondamento e truncamento, comuns em aplicações que trabalham no domínio dos números reais [1].

\section{AUtO-SEQÜÊNCIAS DA TRANSFORMADA NUMÉRICA DE FOURIER}

A seguir, a forma unitária do operador TNF é definida [2].

Definição 1. A transformada numérica de Fourier unitária de x[n], uma sequiência de comprimento $N$ de componentes em $\mathrm{GF}(\mathrm{p})$, é a seqüência $\mathrm{X}[\mathrm{k}], k=0,1, \ldots, N-1$, de elementos em $\mathrm{GF}(\mathrm{p})$ dados por

$$
X[k] \triangleq(\sqrt{N})^{-1}(\bmod p) \sum_{n=0}^{N-1} x[n] \alpha^{k n},
$$

onde $N^{\frac{p-1}{2}} \equiv 1(\bmod p)$ e $\alpha \in G F(p)$ tem ordem $\mathrm{N}$.

H. M. Cavalcanti, R. M. Campello de Souza ${ }_{s}$ Departamento de Eletrônica e Sistemas, Grupo de Processamento de Sinais, Centro de Tecnologia e Geociências, Universidade Federal de Pernambuco, Recife, PE, E-mails: herciliomc@gmail.com, ricardo@ufpe.br.
A transformada inversa de $X[\mathrm{k}]$ é

$$
x[n]=(\sqrt{N})^{-1}(\bmod p) \sum_{k=0}^{N-1} X[k] \alpha^{-k n} .
$$

O par transformado da TNF é denotado por

$$
x[n] \leftrightarrow X[k] .
$$

Algumas propriedades úteis são listadas abaixo.

$\mathrm{P} 1 . \mathrm{X}[\mathrm{n}] \leftrightarrow \mathrm{x}[-\mathrm{k}]$.

$P 2 . E(x[n]) \leftrightarrow E(X[k])$.

P3. $\mathrm{O}(\mathrm{x}[\mathrm{n}]) \leftrightarrow \mathrm{O}(\mathrm{X}[\mathrm{k}])$

onde $\mathrm{E}(\mathrm{x}[\mathrm{n}])$ e $\mathrm{O}(\mathrm{x}[\mathrm{n}])$ denotam, respectivamente, as partes par e ímpar de $\mathrm{x}[\mathrm{n}]$.

$O$ presente trabalho investiga seqüências $x[n]$ cuja TNF $X[k]$ tem a forma

$$
X[k]=\lambda x[k],
$$

$\lambda \in \mathrm{GF}(\mathrm{p})$ ou, em forma de operador, $\Gamma(\mathrm{x}[\mathrm{n}])=\lambda \mathrm{x}[\mathrm{n}]$, onde $\Gamma$ denota o operador TNF unitário. Tais sequiências são então auto-sequências da TNF com autovalor associado $\lambda$ e são denotadas por $\lambda$ N-SITNF. Os valores de $\lambda$ satisfazendo (4) são dados pelo Teorema 1 .

Teorema 1: Os autovalores do operador TNF unitário são as raízes de ordem quatro da unidade $( \pm 1, \pm j)$, onde

$$
j^{2} \equiv-1(\bmod p) \text {. }
$$

Demonstração: Aplicando quatro vezes o operador TNF a $\mathrm{x}[\mathrm{n}]$ e considerando a propriedade P1, obtém-se

$$
\Gamma^{(4)}(x[n])=\lambda^{4} x[k]=x[k],
$$

portanto $\lambda^{4}=1$ e o resultado segue.

É possível provar que [3], se $\mathrm{p}=4 \mathrm{k}+3$ então $\mathrm{j} \in \mathrm{GF}\left(p^{2}\right)$, $\mathrm{k}$ inteiro, e se $\mathrm{p}=4 \mathrm{k}+1$, então

$$
j=\left(\frac{p-1}{2}\right) !(\bmod p) .
$$

O teorema a seguir ilustra um pouco da natureza de uma $\lambda \mathrm{N}$-SITNF.

Teorema 2: Se x[n] é uma $\lambda$ N-SITNF, então $\mathrm{x}[\mathrm{n}]$ é ímpar se $\lambda= \pm \mathrm{j}$ ou par se $\lambda= \pm 1$.

Demonstração: $\mathrm{Se} \mathrm{x}[\mathrm{n}] \leftrightarrow \mathrm{X}[\mathrm{k}]$ é uma $\lambda \mathrm{N}$-SITNF, então utilizando-se a propriedade P1, o par transformado resultante é $\lambda \mathrm{x}[\mathrm{n}] \leftrightarrow \mathrm{x}[-\mathrm{k}]$. Logo $\lambda^{2} \mathrm{x}[\mathrm{k}]=\mathrm{x}[-\mathrm{k}]$, de modo que se $\lambda= \pm 1$ então $\mathrm{x}[\mathrm{n}]=\mathrm{x}[-\mathrm{n}]$ e, se $\lambda= \pm \mathrm{j}, \mathrm{x}[\mathrm{n}]=-\mathrm{x}[-\mathrm{n}]$ e a prova está completa. 
Seqüências ímpares e pares podem ser usadas para gerar $\lambda \mathrm{N}$-SITNFs de acordo com os teoremas 3 e 4 a seguir.

Teorema 3: Se $\mathrm{x}[\mathrm{n}]$ é uma seqüência de comprimento $\mathrm{N}$, então a seqüência $y[n]=E(x[n]) \pm E(X[n])$ é uma $\lambda N$-SITNF com autovalor associado $\lambda= \pm 1$.

Demonstração: Das propriedades P1 e P2 podese obter o par $\mathrm{E}(\mathrm{x}[\mathrm{n}]) \pm \mathrm{E}(\mathrm{X}[\mathrm{n}]) \leftrightarrow \mathrm{E}(\mathrm{X}[\mathrm{k}]) \pm \mathrm{E}(\mathrm{x}[-\mathrm{k}]), \quad \log \mathrm{o}$ $\mathrm{E}(\mathrm{x}[\mathrm{n}]) \pm \mathrm{E}(\mathrm{X}[\mathrm{n}]) \leftrightarrow \pm(\mathrm{E}(\mathrm{x}[\mathrm{k}]) \pm \mathrm{E}(\mathrm{X}[\mathrm{k}]))$ e o resultado segue.

Corolário: Toda seqüência $\mathrm{x}[\mathrm{n}]$ par de comprimento $\mathrm{N}$ gera uma $\lambda N$-SITNF $y[n]=x[n] \pm X[n]$.

Teorema 4: Se $\mathrm{x}[\mathrm{n}]$ é uma seqüência de comprimento $\mathrm{N}$, então a sequiência $\mathrm{y}[\mathrm{n}]=\mathrm{O}(\mathrm{x}[\mathrm{n}]) \mp \mathrm{jO}(\mathrm{X}[\mathrm{n}])$ é uma $\lambda \mathrm{N}$-SITNF com autovalor associado $\lambda= \pm \mathrm{j}$.

Demonstração: Das propriedades P1 e P3, obtémse o par $\mathrm{O}(\mathrm{x}[\mathrm{n}]) \mp \mathrm{jO}(\mathrm{X}[\mathrm{n}]) \leftrightarrow \mathrm{O}(\mathrm{X}[\mathrm{k}]) \mp \mathrm{jO}(\mathrm{x}[-\mathrm{k}])$ ou seja $\mathrm{O}(\mathrm{x}[\mathrm{n}]) \mp \mathrm{jO}(\mathrm{X}[\mathrm{n}]) \leftrightarrow \pm \mathrm{j}(\mathrm{O}(\mathrm{x}[\mathrm{k}]) \mp \mathrm{jO}(\mathrm{X}[\mathrm{k}]))$ e a prova está completa.

Corolário: Toda seqüência $\mathrm{x}[\mathrm{n}]$ ímpar de comprimento $\mathrm{N}$ gera uma $\lambda N$-SITNF $y[n]=x[n] \mp j X[n]$.

\section{SEQÜÊNCIAS EMPREGADAS SOBRE O CANAL SOMADOR REAL}

Os teoremas 3 e 4 ilustram um método simples de se obter $\lambda \mathrm{N}$-SITNFs. Há ainda um outro algoritmo que emprega trigonometria de corpos finitos para gerar famílias de $\lambda \mathrm{N}$ SITNFs para um dado primo da forma $4 \mathrm{k}+3$ [1], [4]. No que se segue, SITNFs são usadas na concepção de um sistema de múltiplo acesso para o canal somador real.

\section{A. Emprego de auto-seqüências da TNF sobre o canal so- mador real}

Um modelo de canal de comunicação bem conhecido é o Canal Somador Real de $\mathrm{t}$ usuários ( $\mathrm{t}-\mathrm{RAC}$ ). No presente trabalho, uma utilização das auto-sequiências da TNF como assinaturas de usuários sobre o 4-RAC é sugerida. No que se segue, $x_{i}[n]$ é a sequiência do usuário i. Essa seqüência deve ser uma $\lambda_{i} \mathrm{~N}$-SITNF, onde $\lambda_{1}=1, \lambda_{2}=-1, \lambda_{3}=\mathrm{j}$ e $\lambda_{4}=-\mathrm{j}$.

\section{A1. 4-RAC}

Seja a seqüência y[n], enviada pelo canal, dada por

$$
y[n]=x_{1}[n]+x_{2}[n]+x_{3}[n]+x_{4}[n] .
$$

Se aplicarmos três vezes a TNF à ambos os membros da equação (8) podemos obter o seguinte sistema de equações, $x_{1}[\mathrm{n}]+x_{2}[\mathrm{n}]+x_{3}[\mathrm{n}]+x_{4}[\mathrm{n}]=\mathrm{y}[\mathrm{n}]$, $x_{1}[\mathrm{n}]-x_{2}[\mathrm{n}]+\mathrm{j} x_{3}[\mathrm{n}]-\mathrm{j} x_{4}[\mathrm{n}]=\Gamma(\mathrm{y}[\mathrm{n}])$, $x_{1}[\mathrm{n}]+x_{2}[\mathrm{n}]-x_{3}[\mathrm{n}]-x_{4}[\mathrm{n}]=\Gamma^{(2)}(\mathrm{y}[\mathrm{n}])$, $x_{1}[\mathrm{n}]-x_{2}[\mathrm{n}]-\mathrm{j} x_{3}[\mathrm{n}]+\mathrm{j} x_{4}[\mathrm{n}]=\Gamma^{(3)}(\mathrm{y}[\mathrm{n}])$, cuja solução leva às expressões de recuperação das sequiências dos usuários, $x_{1}[\mathrm{n}]=\frac{1}{2}(\mathrm{E}(\mathrm{y}[\mathrm{n}])+\mathrm{E}(\mathrm{Y}[\mathrm{n}]))$, $x_{2}[\mathrm{n}]=\frac{1}{2}(\mathrm{E}(\mathrm{y}[\mathrm{n}])-\mathrm{E}(\mathrm{Y}[\mathrm{n}]))$, $x_{3}[\mathrm{n}]=\frac{1}{2}(\mathrm{O}(\mathrm{y}[\mathrm{n}])-\mathrm{jO}(\mathrm{Y}[\mathrm{n}]))$, $x_{4}[\mathrm{n}]=\frac{1}{2}(\mathrm{O}(\mathrm{y}[\mathrm{n}])+\mathrm{jO}(\mathrm{Y}[\mathrm{n}]))$.

\section{B. Exemplos de Auto-seqüências da Transformada Numérica de Fourier.}

A tabela I apresenta exemplos de auto-seqüências da TNF para diversos valores de $\mathrm{p}$ e de $\lambda$.

TABELA I

AUTO-SEQÜÊNCIAS DA TRANSFORMADA NUMÉRICA DE FourIER.

\begin{tabular}{|c||c|c|c|}
\hline$N$ & $\lambda$ & $p$ & seqüência \\
\hline \hline 4 & 1 & 29 & $3,1,1,1$ \\
\hline 4 & -1 & 17 & $15,2,2,2$ \\
\hline 4 & $j=12$ & 29 & $0,28,0,1$ \\
\hline 7 & $j=12$ & 29 & $0,3,9,12,17,20,26$ \\
\hline 7 & $-j=17$ & 29 & $0,9,4,2,27,25,20$ \\
\hline 8 & 1 & 17 & $14,11,15,16,6,16,15,11$ \\
\hline 8 & -1 & 17 & $9,16,7,16,4,16,7,16$ \\
\hline 12 & 1 & 13 & $5,5,4,9,1,1,6,1,1,9,4,5$ \\
\hline 12 & -1 & 13 & $7,8,8,8,8,8,8,8,8,8,8,8$ \\
\hline 12 & $j=5$ & 13 & $0,6,2,11,2,10,0,3,11,2,11,7$ \\
\hline 12 & $-j=8$ & 13 & $0,0,4,5,11,11,0,2,2,8,9,0$ \\
\hline
\end{tabular}

É importante observar que na tabela acima foram considerados apenas primos da forma $4 \mathrm{k}+1$, pois as TNFs obtidas a partir destes primos possuem autovalores reais e inteiros, o que não é possível para a DFT [1]. Esta é uma motivação adicional para a implementação de sistemas multiusuário como o 4-RAC sugerido no presente trabalho.

\section{CONClusões}

Este trabalho investiga seqüências que são invariantes ao operador TNF unitário. Tais seqüências são de relevante interesse, pois o cálculo de suas TNFs é feito com uma complexidade computacional linear. Procedimentos sistemáticos para a obtenção de tais seqüências foram propostos. Um sistema de múltiplo acesso para o canal somador real com quatro usuários, utilizando as auto-seqüências da TNF como assinaturas, foi proposto. Expressões para a recuperação da informação dos usuários foram obtidas. $\mathrm{O}$ desempenho do esquema sugerido está sob investigação.

\section{AGRADECIMENTOS}

Os autores agradecem ao Prof. Dr. Hélio M. de Oliveira por suas valorosas sugestões ao presente trabalho.

\section{REFERÊNCIAS}

[1] R. M. Campello de Souza, H. M. de Oliveira, "Eigensequences for Multiuser Communication over the Real Adder Channel", VI International Telecomunications Symposium (ITS`2006), Fortaleza, Sep. 2006.

[2] J. M. Pollard, "The Fast Fourier Transform in a Finite Field", Math. Comput., vol. 25, No. 114, pp. 365-374, Apr. 1971.

[3] D. M. Burton, "Elementary Number Theory", McGraw-Hill, 4a ed., 1998.

[4] R.M. Campello De Souza, H.M. de Oliveira, A.N. Kauffman, A.J.A Paschoal, "Trigonometry in Finite Fields and a New Hartley Transform", Proceedings of the 1998 IEEE International Symposium on Information Theory, p. 293, Aug. 1998 